\title{
Effects of hypoxaemia and bradycardia on neonatal cerebral haemodynamics
}

\author{
L Nicola Livera, S Andrew Spencer, Maureen S Thorniley, Yapa A B D Wickramasinghe, \\ Peter Rolfe
}

\begin{abstract}
Near infrared spectroscopy has been used to assess the effects of bradycardia and hypoxia on the cerebral circulation in the premature neonate. The technique is well tolerated and can be applied in almost any infant. Continuous monitoring of changes in cerebral oxygenated, deoxygenated, and total haemog. lobin is possible. Total haemoglobin is analogous to cerebral blood volume; thus information on circulatory changes as well as oxygenation state can be obtained. Twenty five babies had cerebral monitoring carried out using this technique. During episodes of hypoxia, both spontaneous and induced, impairment of haemoglobin oxygenation within the brain was detected together with an overall increase in the total mean haemoglobin concentration, which was $0.8 \times 10^{-2}$ $\mathrm{mmol} / 1$. Bradycardia with apnoea also led to impairment of cerebral oxygenation, and to a rapid fall in the concentration of total mean haemoglobin to $1.4 \times 10^{-2} \mathrm{mmol} / \mathrm{l}$, which was followed in some cases by an increase to above the resting value on recovery of the heart rate to a mean of $0.7 \times 10^{-2} \mathrm{mmol} / \mathrm{l}$. These disturbances to total haemoglobin concentration represent abnormalities of cerebral blood volume that may be implicated in the pathogenesis of neonatal cerebral injury.
\end{abstract}

Neonatal cerebral injury is an important cause of morbidity and mortality in the low birthweight and premature infant, and occurs in a significant proportion of neonates despite advances in intensive care techniques. ${ }^{12}$

The aetiology of cerebral injury, both periventricular leukomalacia and periventricular haemorrhage, is not completely understood. Many risk factors have been identified as being associated with the development of cerebral injury,,$^{3-5}$ but recent work suggests that the major underlying mechanism is disturbance to cerebral haemodynamics against the background of a developing brain with immature vasculature and impaired autoregulation. ${ }^{6-8}$ Identification of such disturbance is extremely difficult in the neonate, and at best only single estimates of cerebral blood flow can be made by such methods as ${ }^{133}$ xenon clearance and Doppler ultrasound. ${ }^{10}$ A relatively new technique has been developed, near infrared spectroscopy (NIRS), which appears to have the potential to make continuous, non-invasive measurements of changes in oxygenated and deoxygenated haemoglobin and in total haemoglobin, which is related to blood volume in the tissue being studied.

Previous work by our group in perfused and in situ organs in animals has demonstrated that NIRS is an easily applied technique that can give rapid results in situations that may be changing very quickly. ${ }^{11-13}$ We report here results which show that the technique can also be used to identify abnormalities of the cerebral circulation in infants. The aims of the study were to identify, using NIRS, any disturbances to cerebral blood volume and total haemoglobin and cerebral oxygenation that may occur in association with the commonly seen problems of hypoxia and bradycardia in premature infants.

\section{Methods}

NIRS

The technique of in vivo NIRS was first described by Jobsis, ${ }^{14}$ and its potential value for use in the newborn was reported by Rea et al. ${ }^{11}$ Brazy et al also described its application in the neonate. ${ }^{15}$ Tissue is transilluminated by light in the near infrared region $(700-1000 \mathrm{~nm})$, which penetrates tissue much more readily than visible light. Certain chromophores within tissue show specific absorption characteristics, in particular oxygenated and deoxygenated haemoglobin.

Using several specific wavelengths in the near infrared part of the spectrum it is possible to monitor these chromophores within the brain. ${ }^{11} 16$ The derivation of quantitative determinations is, however, less than straightforward. Algorithms that utilise the Beer-Lambert law and incorporate derived extinction coefficients for oxygenated and deoxygenated haemoglobin are used to convert changes in optical absorbance, A, to changes in concentration of the chromophores being studied. Near infrared multiplier coefficients for calculation of concentration changes for the three wavelengths used are given in the table. ${ }^{17}$ For example the change in concentration of deoxygenated haemoglobin is given as: A1 (1.641)-A2 $(0.935)-A 3(0.178)$ where $A 1, A 2$, and $A 3$ are the changes in absorbance at 775,845 , and 904 $\mathrm{nm}$ respectively.

Near infrared multiplier coefficients

\begin{tabular}{llrr}
\hline Haemoglobin & \multicolumn{4}{l}{ Wavelength $(\mathrm{nm})$} & \\
\cline { 2 - 4 } & \multicolumn{1}{l}{} & \multicolumn{1}{c}{845} & \multicolumn{1}{c}{904} \\
\hline Oxygenated & -1.156 & 0.074 & 1.428 \\
Deoxygenated & 1.641 & -0.935 & -0.178 \\
Total & 0.485 & -0.861 & 1.249 \\
\hline
\end{tabular}

Total
Correspondence to: Dr Livera.

Accepted 19 September 1990 


\section{INSTRUMENTATION}

NIRS instrumentation has been developed by the department of biomedical engineering and medical physics, University of Keele. The instrument uses four laser diodes of wavelenghts 775, 805, 845, and $904 \mathrm{~nm}$. The laser firing circuit is controlled by a microprocessor and provides light pulses of $200 \mathrm{~ns}$ duration that are transmitted along flexible glass optical fibres. Analysis of light transmitted through the biological organ is then carried out by computer using the near infrared multiplier coefficients as described above. Changes in concentration of oxygenated and deoxygenated haemoglobin and in total haemoglobin are displayed in real time. ${ }^{11} 1218$ The system achieves a coefficient of variation of $0.01 \%$ over a 30 second period while monitoring a basal level of eight optical density units. System drift is less than 0.004 optical density/hour for all four laser diodes.

The light energy produced falls well within safety limits as set by the British Standards Institute (BSI 4803). The near infrared system produces 0.133 Joules $(\mathrm{J})$ per square metre (for single pulse) and 173 Watts (W) per square metre (mean power) compared with BSI limits of $200 \mathrm{~J}$ (single pulse) and $2000 \mathrm{~W}$ (mean power). A sensation of warmth occurs at 1000 W. No adverse effects of NIRS have been reported.

\section{NEONATAL APPLICATION}

For studies on the neonatal brain near infrared light at the appropriate wavelengths is transmitted along glass fibre optic bundles, through an attachment probe in which the light is turned through 90 degrees, thus enabling the fibres to rest alongside the baby's head. Double sided adhesive rings are used to keep the probes in place on opposite sides of the head on the temples. The interoptrode distance is measured with calipers between the surface of the two attachment devices. They are then bandaged in place. This is well tolerated by most infants, and other nursing and medical care can continue. The probes incorporate a safety switch that automatically stops the transmission of light if the probes become detached from the baby's head, in order to prevent the accidental entry of light into the eye.

\section{QUANTITATIVE MEASUREMENTS}

All values given are for changes in concentration from a baseline value during stable periods. In order to carry out absolute quantitation of results the determination of optical pathlength is required. As tissue is a complex, highly scattering medium, the optical pathlength is greater than the physical separation of transmitter and detector. Current estimates of optical pathlength give a value of approximately 4.5 times the interoptrode distance, ${ }^{19}$ but it is generally accepted that further work is required in this area. Therefore no attempt is made here to measure absolute values because of the many assumptions required to do this and the remaining uncertainties regarding actual optical path- length. In all plots shown the $y$ axis represents change in concentration in $\mathrm{mmol} / \mathrm{l}$ for the whole sample being studied. In order to make some comparisons between different babies (with different sized samples or heads), results are expressed per $\mathrm{cm}$ of sample size. This is derived from the physical interoptrode distance; the total change in concentration being divided by this figure to give change in concentration per $\mathrm{cm}$ of brain. The assumption is thus made that there is an association between optical pathlength and physical pathlength, which does not vary greatly with gestation.

Changes in the concentration of total haemoglobin are of great importance as they are considered to represent changes in blood volume of the sample being studied. In the brain all haemoglobin is contained within the vascular compartment unless haemorrhage has occurred. Provided that packed cell volume and therefore the association between plasma volume and haemoglobin remains constant, then changes in the concentration of total haemoglobin will reflect changes in total cerebral blood volume. For studies over a fairly short time period the packed cell volume can be assumed to be constant.

In order to express changes in haemoglobin oxygenation, the haemoglobin oxygenation index (oxygenated minus deoxygenated) is used. This enables comparison between babies and can be used in circumstances where there is a change in total haemoglobin.

\section{Patients}

(A) HYPOXIA STUDY

Sixteen infants with a total of 50 episodes of hypoxia were studied. These infants were all recovering from respiratory distress syndrome or had bronchopulmonary dysplasia and were still oxygen dependent at the time of study. Gestational age varied from 24 to 33 weeks (median 29), birth weight ranged from 580 to $2720 \mathrm{~g}$ (median 1012), and age at the time of study was from 3 to 105 days (median 20).

The babies were all stable in low concentrations of oxygen, and had normal cranial ultrasound scans or minimal abnormalities.

These babies were studied during spontaneous $(n=20)$ or induced $(n=30)$ falls in arterial saturation as measured by pulse oximetry (using an Ohmeda Biox $3700 \mathrm{e}$ ). A change in arterial saturation of 5 to $10 \%$ was induced by altering the inspired oxygen for a period of five minutes, then returning this to the initial value. This change is considered safe, and is similar to that seen during nursing procedures and feeds. In those infants monitored during spontaneous fluctuations in arterial saturation the concentration of inspired oxygen was increased if the saturation fell below $80 \%$.

In three babies also being monitored with transcutaneous carbon dioxide electrodes no significant change in skin carbon dioxide tension was seen, but it is possible that a transient brief rise in arterial carbon dioxide tension occurred. Continuous blood pressure data were not available for these studies as all indwelling lines had been removed by the time of study. 
(B) BRADYCARDIA STUDY

In a second group of nine babies, a total of 15 studies were carried out during spontaneous bradycardias. In this group gestation varied from 24 to 30 weeks (median 27), birth weight from 580 to $1120 \mathrm{~g}$ (median 990), and age at the time of study from 3 to 96 days (median 20). The babies were either recovering from respiratory distress syndrome or had bronchopulmonary dysplasia, and they were all relatively stable in headbox oxygen. The bradycardias in all cases were considered to be related to apnoea of prematurity and not symptomatic of other illness-that is, infection, anaemia, etc, had been excluded. In some infants treatment with oral theophyllines had been started.

Monitoring was carried out during bradycardias with spontaneous recovery; if intervention was required then monitoring was terminated and the baby stimulated as appropriate.

As in the previous protocol, only three babies were of an age where transcutaneous carbon dioxide monitoring could be carried out. No change in skin carbon dioxide tension with bradycardia was detected, but again it is possible that arterial carbon dioxide tension changed in some infants. Direct blood pressure monitoring was not possible.

Approval from the local ethical committee was given for both studies and informed parental consent was obtained for each baby.

\section{Results}

Background noise while monitoring a baby in a stable state at rest was found to be \pm 0.0027 $\mathrm{mmol} / \mathrm{l}$ for deoxygenated and \pm 0.0026 mmol/l for oxygenated and total haemoglobin.

\section{(A) HYPOXIA STUDY}

For babies studied during changes in arterial saturation, it was found that a fall in arterial oxygen saturation $\left(\mathrm{SaO}_{2}\right)$ was consistently associated with a change in oxygenation index as monitored by NIRS. The size of the fall was related to the degree of change in oxygenation index as can be seen in fig 1 . In the majority of cases total haemoglobin (oxygenated plus deoxygenated), increased during arterial desaturation (mean $0.8 \times 10^{-2} \mathrm{mmol} / 1$ of brain, SEM $0.7 \times^{10-3}$ ) (fig 2). No association was demonstrated between the change in total haemoglobin and the degree of desaturation. Figure 3 shows the results of a typical study on a baby of $\mathbf{3 0}$ weeks' gestation at age 5 weeks, during a period of induced desaturation. A rapid rise in deoxygenated and fall in oxygenated haemoglobin is seen as soon as the $\mathrm{SaO}_{2}$ starts to fall, and both return to initial values as the saturation recovers. Total haemoglobin concentration shows a small increase with desaturation.

\section{(B) BRADYCARDIA STUDY}

The second group of babies were studied during bradycardic episodes accompanied by a fall in $\mathrm{SaO}_{2}$. In all cases there was a fall in total haemoglobin immediately after the bradycardia (mean (SEM) $1.4(0.16) \times 10^{-2} \mathrm{mmol} / \mathrm{l}$ of brain) (fig 2). Again in all cases as the bradycardia recovered to the original resting heart rate the total haemoglobin concentration increased up to the previous value. In nine cases total haemoglobin continued to increase to a concentration above the previous resting value after the bradycardia had recovered (mean (SEM) 0.7 $(0.07) \times 10^{-2} \mathrm{mmol} / 1$ of brain) (fig 2).

Typical results from a baby studied during an episode of bradycardia are shown in fig 4. In this example total haemoglobin increased above the initial value after recovery of the bradycardia. The effects of multiple bradycardias in an infant of 28 weeks' gestation are shown in fig 5. The fluctuations in total haemoglobin and thus cerebral blood volume can be seen to follow closely the heart rate variation during these episodes.

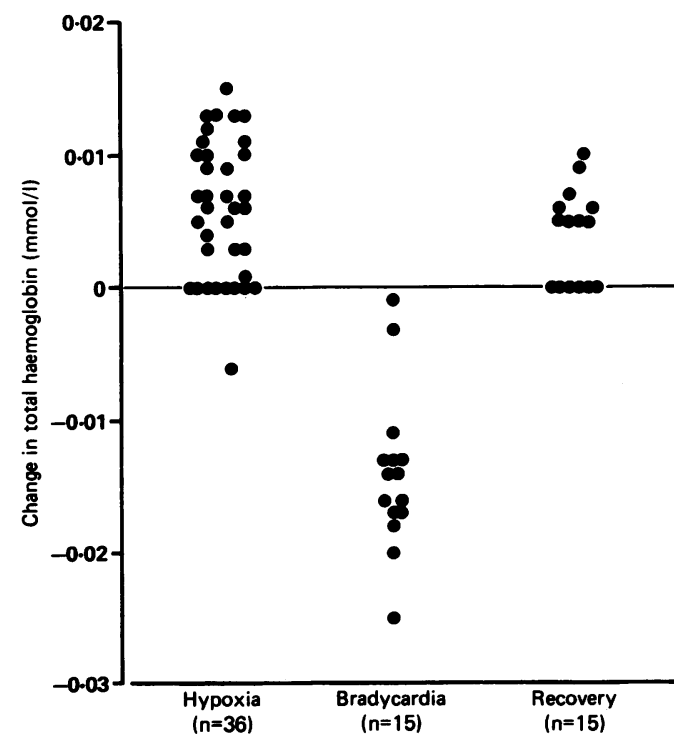

Figure 2 Changes in total cerebral haemoglobin in 36 episodes of hypoxia in 12 infants and 15 episodes of bradycardia with subsequent recovery in nine infants.
Figure 1 The association between change in $\mathrm{SaO}_{2}$ and change in haemoglobin oxygenation index for 48 episodes of spontaneous and induced desaturation.

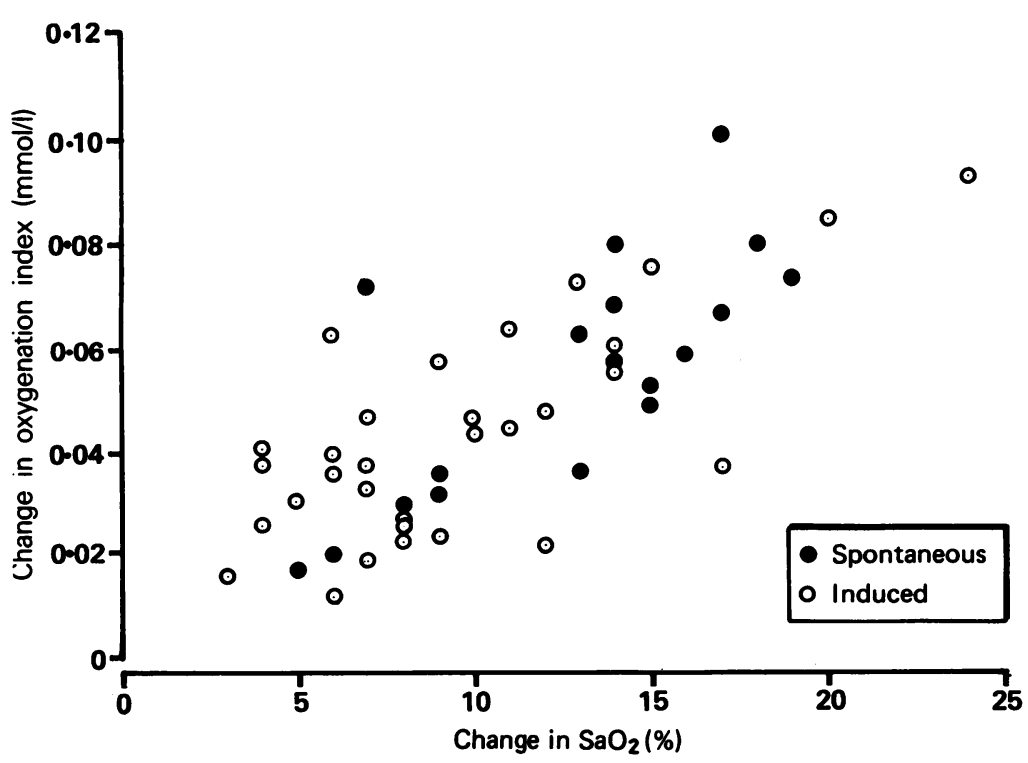




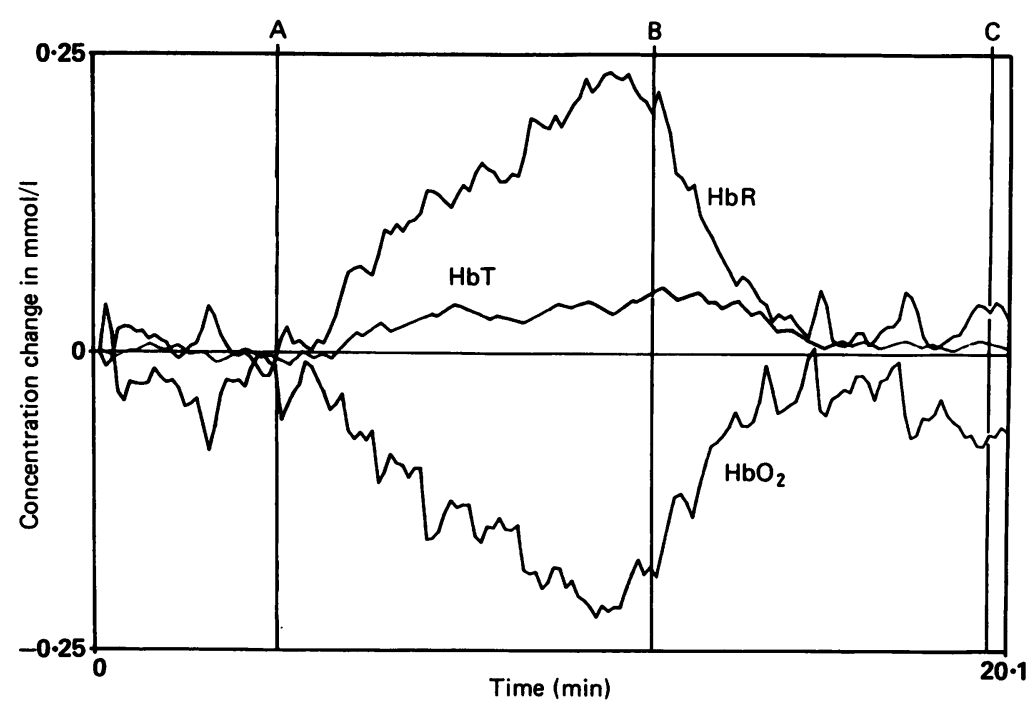

Figure 3 The effects of hypoxia on the concentrations of total $(H b T)$, deoxygenated $(H b R)$ and oxygenated haemoglobin $\left(\mathrm{HbO}_{2}\right)$ in a preterm infant of 30 weeks' gestation. Arterial saturation gradually falls from $94 \%$ at $(A)$ to a lowest value of $83 \%$ at $(B)$, then rises to $93 \%$ at $(C)$.

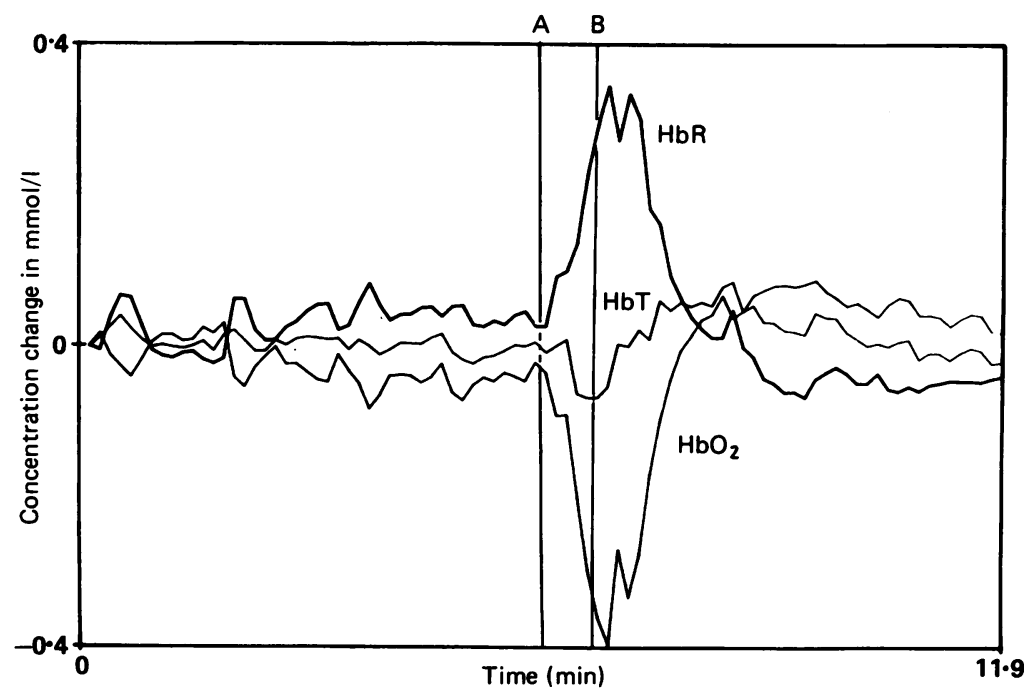

Figure 4 Changes in the concentrations of total $(H b T)$, deoxygenated $(H b R)$ and oxygenated haemoglobin $\left(\mathrm{HbO}_{2}\right)$ in a preterm infant of 27 weeks' gestation in response to bradycardia at $(A)$. Heart rate fell from $144 \mathrm{bpm}$ at $(A)$ to $68 \mathrm{bpm}$ at $(B)$ with prompt recovery; arterial saturation fell from $91 \%$ at $(A)$ to $60 \%$ at $(B)$.

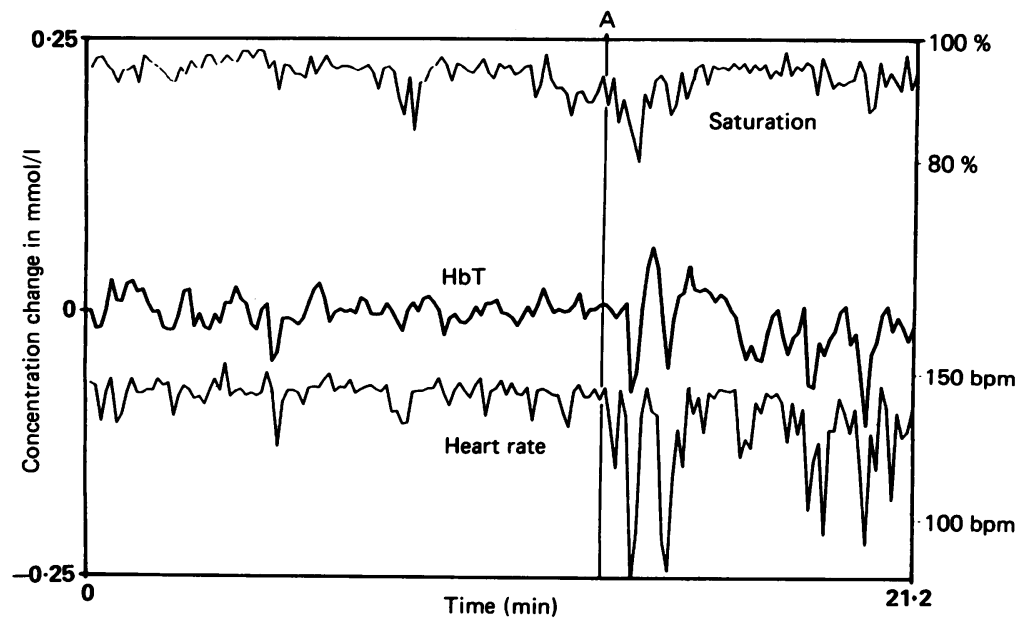

Figure 5 Changes in total haemoglobin (HbT), heart rate, and $\mathrm{SaO}_{2}$ during multiple bradycardias beginning at $(A)$, in an infant of 28 weeks' gestation.
Cumulative data on all the babies studied, indicating the change in total haemoglobin occurring either during bradycardia and recovery or during a saturation change, are shown in fig 2. These demonstrate that though the degree of change in total haemoglobin occurring during bradycardias was greatest, the order of magnitude for these changes was similar for all studies. In four cases the changes could not be analysed because of movement artefact.

\section{Discussion}

These studies have demonstrated that in most cases arterial desaturation of a moderate degree is associated with an increase in cerebral total haemoglobin, whereas bradycardia is associated with a fall in cerebral total haemoglobin, and in some instances with an overshoot on recovery.

Direct comparisons of results from babies of different gestation can raise some theoretical difficulties. The interoptrode distance for the babies studied was in the range 5.3 to $8 \mathrm{~cm}$. Although the current estimate of optical pathlength is approximately 4.5 times the interoptrode distance, the use of this factor in babies of different gestational ages and weights, as well as the extent of variations in light scattering with differing stages of cerebral development, is yet to be fully evaluated.

Total haemoglobin concentration is related to cerebral blood volume under circumstances when haemoglobin and packed cell volume are remaining relatively constant, as in these studies over a fairly short time period. Thus changes in total haemoglobin during such studies represent changes in cerebral blood volume in association with specific clinical situations.

The most striking finding is of the fall in volume with bradycardia. In the neonate cardiac output is very dependent on heart rate, so a fall in heart rate would be expected to lead to a fall in cardiac output, cerebral blood flow, and thus cerebral blood volume. ${ }^{20}$ Due to the technical difficulties discussed earlier in studying such parameters in babies, such expected changes have been difficult to demonstrate. Cooke et al, using jugular venous occlusion plethysmography, demonstrated a fall in estimated cerebral blood flow with fall in heart rate followed by an increase to above the resting level in two babies. ${ }^{21}$ Some workers have suggested that as mean arterial blood pressure appeared to be maintained by a decrease in peripheral blood flow during apnoea and bradycardia, cerebral blood flow was likely to be maintained also. ${ }^{22}$ In a study using Doppler techniques to measure cerebral blood flow velocity, however, both diastolic and systolic blood flow velocity decreased with bradycardia of less than 80 beats per minute. ${ }^{23}$ The authors suggested that these changes, which paralleled similar changes in systemic blood pressure, would support the concept of a pressure passive cerebral circulation (that is, lack of autoregulation). These results are in accord with those found in our studies using NIRS. It would appear from these that cerebral blood volume is closely related to heart rate at least during single and multiple bradycardias. 
Bradycardias and apnoeas in infants are usually treated vigorously but are very common in the premature baby. Although most respond rapidly to appropriate treatment, there are some babies who continue to have fairly frequent episodes. Such abnormalities of heart rate also occur during ventilation-for example, with suction, tube blockage, etc. Acute falls in cerebral blood volume, particularly if repeated, may well be one mechanism leading to ischaemia in areas at risk because of their immature blood supply. This could result in cerebral infarction-that is, periventricular leukomalacia.

In some babies an overshoot in total haemoglobin and cerebral blood volume is seen after a bradycardia. The reason for this is not clear. Although no significant change in transcutaneous carbon dioxide measurement was observed in those babies being monitored in this way, a rise in arterial carbon dioxide tension as a result of apnoea in association with the bradycardia could well lead to this phenomenon. It may also represent reactive vasodilatation due to hypoxia and acidosis induced by the bradycardia and apnoea. ${ }^{24}$ Also of interest is the increase in total haemoglobin seen in some cases with desaturation. This may represent vasodilatation in response to even mild hypoxia. These results tend to suggest that the technique of using a small change in $\mathrm{SaO}_{2}$ to calculate absolute cerebral blood volume, assuming that the latter does not change during the manoeuvre, ${ }^{25}$ may be inaccurate.

\section{CONCLUSIONS}

Cerebral circulatory instability and abnormalities have been demonstrated in association with common neonatal problems and give further insight into the pathogenesis of ischaemic damage. NIRS has been shown to be capable of detecting such abnormalities non-invasively.

We thank the staff of the neonatal unit for their help and all the parents who consented to these studies being carried out. This parents who consented to these studies being carried out. This work was supported by the North Staffordshire Medical Insti-
tute, West Midlands Regional Health Authority, and the Science tute, West Midlands Regional Health

1 Cooke RWI. Early and late ultrasonographic appearances and outcome in very low birth weight infants. Arch Dis Child 1987;62:931-7.

2 Marlow N, D'Souza SW, Chiswick ML. Neurodevelopmental outcome in babies weighing less than $2001 \mathrm{~g}$ at mental outcome in
birth. $B M \mathcal{Y} 1987 ; 294: 1582-4$.
3 Calvert SA, Hoskins EM, Fong KW, Forsyth SC. Aetiological factors associated with the development of periventricu-

lar leukomalacia. Acta Paediatr Scand 1987;76:254-9.

Trounce JQ, Shaw DE, Levene MI, Rutter N. Clinical risk factors and perive

5 Miall-Allen VM, de Vries LS, Whitelaw AGL. Mean arterial blood pressure and neonatal cerebral lesions. Arch Dis Child 1987;62:1068-9.

6 Perlman JM, Mcmenamin JB, Volpe JJ. Fluctuating cerebral blood flow velocity in respiratory distress syndrome. N Engl f Med 1983;309:204-9.

7 Ment LR, Duncan CC, Ehrenkranz RA, et al. Intraventricular haemorrhage in the preterm neonate: timing and

8 Lou HC Perinat changes. F Pediat 1984;104:419-25. intraventricular haemorrhage; a pathogenetic model. Arch Neurol 1980;37:585-7.

9 Greisen G. Cerebral blood flow in preterm infants during the

10 Levene MI, Cerebral Blood Flow. Neonatal neurology. (Current reviews in paediatrics.) Edinburgh: Churchill Current reviews in paedi

11 Rea PA, Crowe J, Wickramasinghe Y, Rolfe P. Non-invasive optical methods for the study of cerebral metabolism in the human newborn: a technique for the future? $\mathcal{f}$ Med Eng Technol 1985;9:160-6.

12 Wickramasinghe YABD, Crowe J, Rolfe P. Near infrared techniques for monitoring metabolism and blood oxygen. Proceedings of the 8th Annual Conference of the Institute of Electrical and Electronic Engineers Engineering in Medicine and Biological Sciences. Dallas, Fort Worth, 1986:1172-4.

13 Thorniley MS, Wickramasinghe YABD, Rolfe P. Monitoring cerebral tissue oxygenation non-destructively in the rat and rabbit brain. Biochem Soc Trans 1988;16:980-2.

14 Jobsis FF. Non-invasive infra red monitoring of cerebral and myocardial oxygen sufficiency and circulatory parameters. myocardial oxygen sufficienc.
Science 1977;198:1264-6.

15 Brazy JE, Lewis DV, Mitnick MH, Jobsis FF. Noninvasive monitoring of cerebral oxygenation in preterm infants: monitoring of cerebral oxygenation in preterm infants:

16 Wray S, Cope M, Delpy DT, Wyatt JS, Reynolds EOR. Characterisation of the near infra red absorption spectra of Characterisation of the near infra red absorption spectra of
cytochrome aa 3 and haemoglobin for the non-invasive cytochrome aa3 and haemoglobin for the non-invasive
monitoring of cerebral oxygenation. Biochim Biophys Acta monitoring of cere

17 Wickramasinghe YABD, Thorniley M, Rolfe P, Livera LN, Spencer SA. Development of algorithms for in-vivo noninvasive monitoring in biological tissue using near infra red spectroscopy. Proceedings of the 5 th Mediterranean Conference on Medical and Biological Engineering. Patras, 1989:252-3.

18 Thorniley MS, Livera LN, Wickramasinghe YABD, Rolfe P. Non-invasive monitoring of cerebral tissue oxygenation in-vivo by near infra red spectroscopy: a sensitive indicator in-vivo by near infra red spectroscopy: a sensitive indicator

19 Delpy DT, Cope M, van der Zee P, Arridge S, Wray S, Wyatt JS. Estimation of optical pathlength through tissue from direct time of flight measurement. Phys Med Biol from direct time

20 Hatch DJ. Paediatric anaesthesia. In: Nimmo WS, Smith G, eds. Anaesthesia. Oxford: Blackwell Scientific Publications, eds. Anaesthesia

21 Cooke RWI, Costeloe K, Rolfe P, Tizard JPM. Measurement of cerebral blood flow in the newborn. In: Stern L, Oh W, Friis-Hansen B, eds. Intensive care in the newborn. 2nd Ed. New York: Masson Publishing, 1978:127-33.

22 Storrs CN. Cardiovascular effects of apnoea in preterm infants. Arch Dis Child 1977;52:534-40.

23 Periman JM, Volpe JJ. Episodes of apnea and bradycardia in the preterm newborn: impact on cerebral circulation. Pediatrics 1985;76:333-8.

24 Lassen NA. The luxury perfusioin syndrome and its possible relation to acute metabolic acidosis localised within the brain. Lancet 1966;ii:1113-5.

25 Wyatt JS, Cope M, Delpy DT, Wray S, Reynolds EOR. Quantification of cerebral oxygenation and haemodynamics in sick newborn infants by near infrared spectrophotometry. Lancet 1986;ii:1063-6. 